MADGU. Mundo, Arquitectura, Diseño gráfico y Urbanismo. NÚM 08, 2021 ISSN: 2594-1208, PP. 26-47

Recibido: 16 de Abril de 2021

Aceptado: 12 de Mayo de 2020

DOI: https://doi.org/10.36800/madgu.v4i8.67
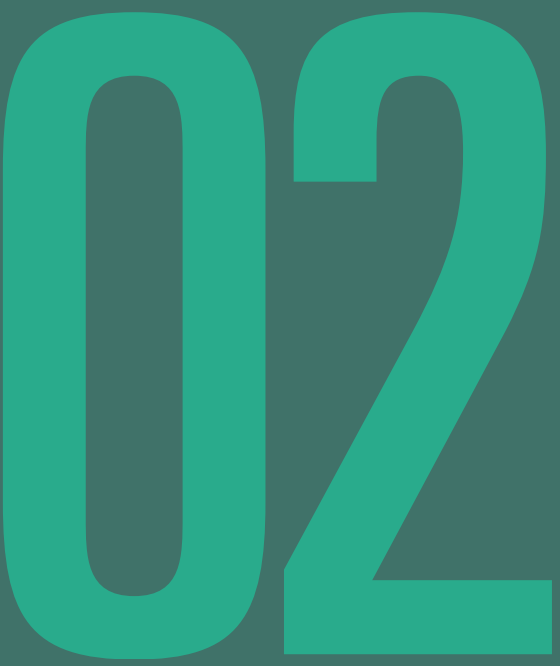

\title{
IMPLEMENTACIÓN DE DISEÑO CONTEXTO-ENTORNO: ESTUDIO ECOCÉNTRICO EN PARADAS DE AUTOBÚS
}

\section{Implementation of Context-Environment Design: Ecocentric Study in Bus Stops}

Sofía Alejandra Luna Rodríguez sofia.lu-nard@uanl.edu.mx https://orcid.org/0000-0003-2879-4132 Universidad Autónoma de Nuevo León, Diseñadora Industrial doctora en Arquitectura Diseño y Urbanismo (UAEM). Profesora de la Facultad de Arquitectura en la Licenciatura en Diseño Industrial de la Universidad Autónoma de Nuevo León.

Francisco Javier Godino Peña franciscogod97@gmail.com Diseñado Industrial por la Universidad Autónoma de Nuevo León Fundador y Director General de Algosa

Servicios Integrales. 
Palabras clave: Parada de autobús, ecocentrismo, diseño, sostenible Keyword: Bus stop, ecocentrism, design, sustainable.

\section{Resumen:}

Hoy en día, las teorías de diseño antropocéntricas explican que el lado empático y centrado en el usuario han sido parte fundamental en la creación de productos, servicios y sistemas a lo largo de los años. Sin embargo, los resultados no logran ser lo suficientemente competentes para considerar factores externos al usuario como ambientes naturales, contextos externos al humano, tendencias y relaciones con la futurología. Es por esto que implementando enfoques más amplios y considerando aspectos valiosos como la biología, sociología, estudio de tendencias, antropología y medio ambiente se puede lograr, junto con el factor humano-empático, un resultado de diseño completo.

Este proyecto nace de la inquietud de aplicar nuevos enfoques de métodos de diseño para la creación de productos, espacios y sistemas que conviven en la vida cotidiana del ser humano, a través de una metodología basada en el Contexto-Entorno llamada "Generación de ideas orientadas a los ecosistemas", en este caso, para una parada de autobús en la Cd. de Monterrey, Nuevo León, México.

El concepto de diseño seleccionado presenta las características relevantes definidas a partir de la generación de ideas orientadas a los ecosistemas y cumpliendo con la Norma Técnica Estatal de Aceras de Nuevo León, permitiendo un diseño completo a partir de los objetivos del contexto al cual va dirigido.
Abstract:

Nowadays, anthropocentric design theories explain that the empathic and user-centered side has been a fundamental part in the creation of products, services, and systems over the years, however, the results do not manage to be sufficiently competent to consider external factors to the user such as natural environments, contexts external to the human, trends, and relationships to futurology. This is why implementing broader approaches and considering valuable aspects such as biology, sociology, the study of trends, anthropology, and the environment, together with the human-empathic factor, a complete design result can be achieved.

This project was born from the concern to apply new approaches to design methods for the creation of products, spaces, and systems that coexist in the daily life of the human being, through a methodology based on the context-environment called "Generation of oriented ideas to ecosystems", in this case, for a bus stop in the City of Monterrey, Nuevo León, Mexico.

The selected design concept presents the relevant characteristics defined from the generation of ideas oriented to ecosystems and complying with the State Technical Standard for Sidewalks of Nuevo León, allowing a complete design based on the objectives of the context to which it is directed. 
Dra. Sofía Alejandra Luna Rodríguez

Francisco Javier Godino Peña

\section{DISEÑO CONTEXTO-ENTORNO: DISEÑANDO A TRAVÉS DEL ECOCENTRISMO}

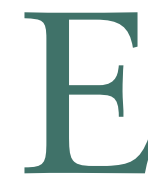

1 diseño está en el borde de una cuarta Revolución Industrial: la Era Biológica (Oxman, 2016). Neri Oxman, fundadora del grupo de investigación Mediated Matter del MIT Media Lab, establece que, debido al momento tecnológico en el que vivimos ahora, estamos entrando en la Era Biológica de la industria y el diseño. La Era Biológica significa la convergencia entre la tecnología y la naturaleza para mejorar el diseño, la arquitectura y el diseño unido al entorno natural.

El ecocentrismo es un concepto basado en el desarrollo sostenible. Este concepto sugiere que las acciones y los pensamientos del individuo deben centrarse en el medio ambiente por sobre todas las cosas, tanto en su cuidado como en la conservación. 
El contexto se introdujo por primera vez en la investigación de diseño por el teórico en diseño Christopher Alexander en su libro Notas sobre la síntesis de la forma (1964). Alexander menciona que el contexto (entorno que rodea) define el problema de diseño en torno a la solución como forma (objeto). A partir de ello, el contexto influye en las propuestas de diseño, formando un diseño guiado por el contexto.

El contexto de un producto, donde estará implementado, y la relación de los parámetros externos con los que interactúa influyen en gran medida con la función, forma y tamaño del mismo producto, tal como lo mencionó Gustavo Zepeda (2017):

El contexto puede cambiar fácilmente el resultado de un diseño por la relación con los elementos con los que interactúa, variables que influencian como el entorno, entre otros. Por lo tanto, podemos entender que, la forma de un objeto, varía significativamente dependiendo del contexto en que está (p.656).

La gran influencia del contexto nativo, dígase ecosistemas naturales o entornos creados por el humano que existen en una parada de autobús, es, en gran medida, detonante de la forma y función de dichas paradas. Es por eso que se utilizará la metodología basada en el contexto, para así poder materializar las necesidades y resolver las problemáticas que los usuarios tienen al momento de utilizar una parada de autobús en la ciudad de Monterrey.

Con base en esta metodología definiremos 4 contextos principales:

- Contexto innovativo: la modernización de las cosas y cambios posibles e inexistentes o, como menciona Zepeda (2017), “subjetivas” de aspectos políticos, tecnológicos, económicos y sociales.

- Contexto nativo: entornos físicos naturales o creados por el hombre que influenciarán directamente en la parada de autobús.

- Contexto humano: factores sociales a gran escala, pasando por antropología, opiniones y el diseño centrado en el usuario. 
- Contexto de diseño: entrelazando los contextos anteriores se logra un equilibrio de todos estos llegando a una solución total que da como resultado una parada de autobús funcional y destinada específicamente al entorno establecido.

Existen 6 fases para la implementación de esta metodología, de las cuales se ven complementadas con otras herramientas de diseño como se observa en la Figura 1.

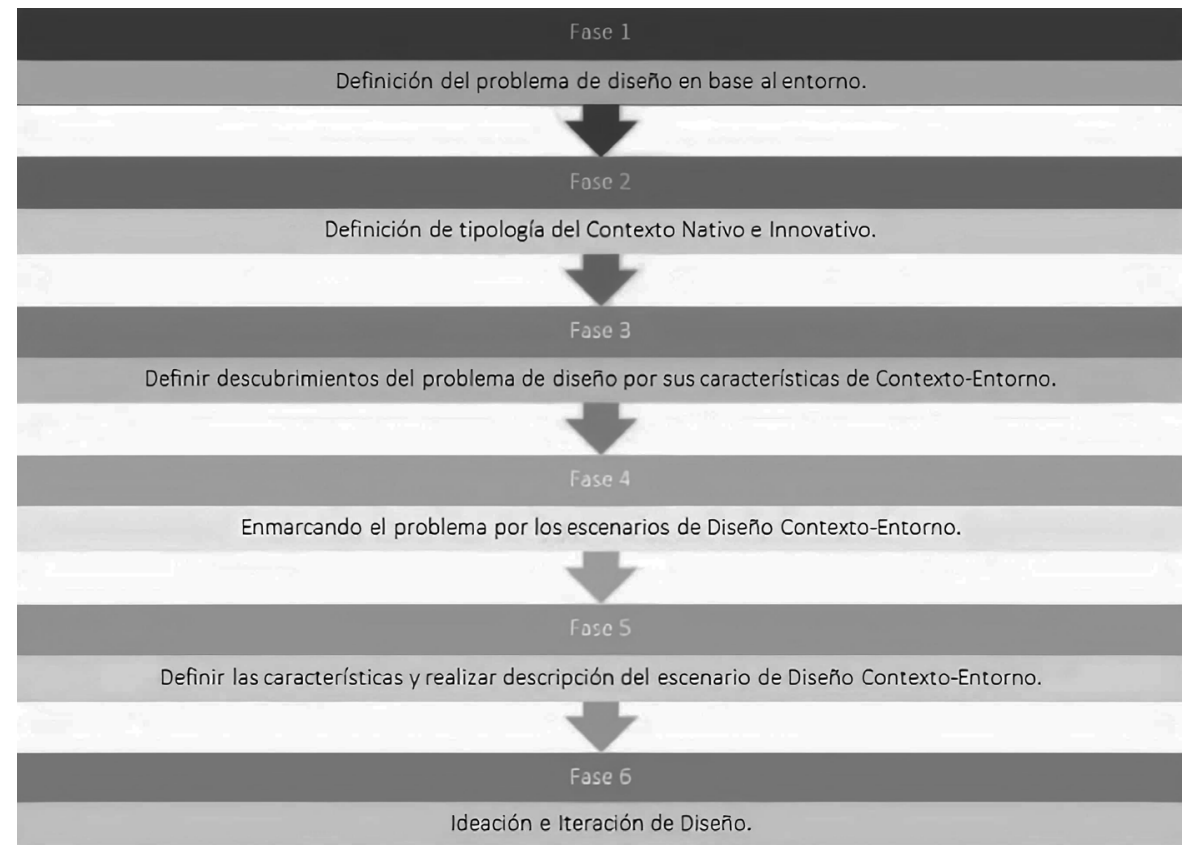

Figura 1. Fases de escenarios en Contexto-Entorno. Adaptado de (Zepeda, 2017, p.655).

El contexto y entorno siempre han sido de gran relevancia para el diseño y, aunque es aún un campo poco explorado, busca la mejor forma de resolver una problemática general o específica y que siempre esté creado y fabricado para su ambiente, tal y como dijo Wucius Wong (2002):

En pocas palabras, un buen diseño es la mejor expresión visual de la esencia de “algo", ya sea esto un mensaje o un producto. Para hacerlo fiel y eficazmente, el diseñador debe buscar la mejor forma posible para que ese "algo" sea conformado, fabricado, distribuido, usado y relacionado con su ambiente (p. 9). 


\section{FASE 1: DEFINICIÓN DEL PROBLEMA CON BASE EN EL EN- TORNO}

Para poder definir los problemas que existen en el entorno se tomó como base la observación y análisis de caso en una localización establecida para el prototipo de la parada de autobús. De este se reconocieron varios factores que influyen en el entorno, tales como la demografía peatonal; la gran concentración de rutas urbanas y camiones, debido a la densidad de personas alojadas en este sitio determinado; el clima árido y carente de humedad de la Cd. de Monterrey y, por supuesto, se consideraron aspectos sociales y físicos de diversos lugares del municipio de Monterrey. Estos factores dieron pie a proponer la realización de un análisis de sitio enfocado en las temperaturas promedio de la ciudad, asoleamientos producidos en el entorno a trabajar y tomando en cuenta la influencia de los vientos dominantes y reinantes.

Con base en estos lineamientos se realizó un censo vehicular para conocer la frecuencia y cantidad de camiones urbanos del sitio, el nivel socioeconómico del entorno y el nivel demográfico, tomando como referencia un radio de 3 $\mathrm{km}$, condiciones de la banqueta y accesibilidad al tomar los camiones.

En 2015 el Gobierno de Nuevo León realizó un estudio detallado donde se implementaría un desarrollo para la accesibilidad universal; en él incluiría la ampliación de banquetas, reducción de velocidades de los vehículos, semaforización y colocación de mobiliario urbano para personas mayores de edad o con alguna discapacidad. Hoy en día, dicho proyecto ya ha sido implementado en varias calles de la Cd. de Monterrey, dando como resultado la calle Juan I. Ramón y entrecruces aledaños. Por este motivo se prefirió como sitio de estudio el cruce de la Av. Benito Juárez y la calle Juan I. Ramón (Figura 2).

\section{CENSO DE CAMIONES URBANOS}

El censo se realizó el Domingo 02 de febrero de 2020 entre las 13:05 horas con una duración de 20 min cada censo tomando periodos de descanso de 15 min, dando un total de 3 censos. Los resultados arrojaron que en promedio transitan un total de 174 camiones urbanos por hora y que se conforman con más de 18 rutas distintas teniendo un gran rango de traslado para los usuarios. 
Problemática preliminar: Gran demografía peatonal y aglomeración de camiones urbanos

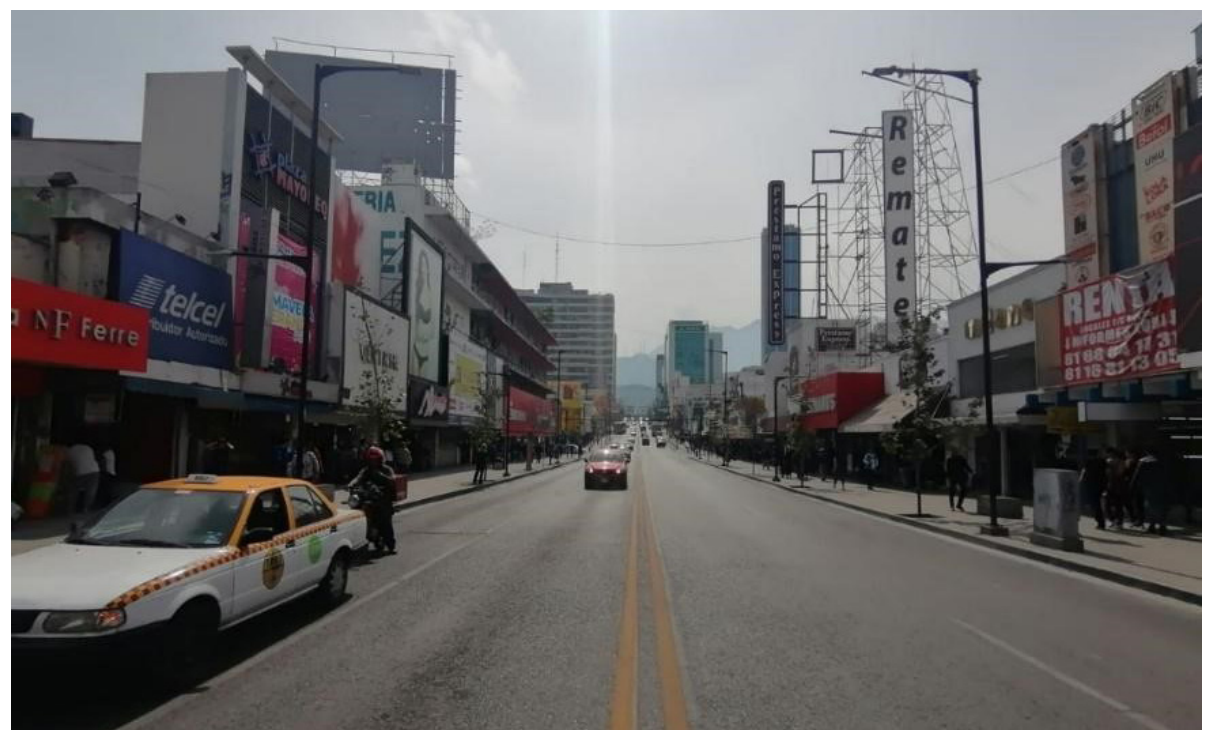

Figura 2. Av. Benito Juárez y calle Juan I. Ramón. Calle Benito Juárez y la calle Juan I. Ramón (Fotografía: Godino, 2019).

\section{FASE 2: DEFINICIÓN DE TIPOLOGÍA DE CONTEXTO NATI- VO E INNOVATIVO}

\section{CONTEXTO NATIVO}

Se comienza con lo que ya está implementado en la intersección de la calle Benito Juárez y la calle Juan I. Ramón actualmente, para luego poder pasar a la parte subjetiva o lo que podría aplicarse en el entorno en un futuro. Partiendo desde la observación, existe un número limitado de paradas de autobuses, a pesar de haber un gran número de peatones esperando por periodos cortos de tiempo para tomar la ruta de camión deseada. Así mismo, las calles cuentan con un amplio espacio; sin embargo, este se convierte en espacio muerto, ya que no existen áreas verdes que puedan ayudar a la percepción del espacio.

Según el Sistema Integral de Transporte Metropolitano (SITME), existen 4,962 rutas oficiales transitando actualmente por toda el área metropolitana de Monterrey, las cuales se dividen como: 
- Rutas radiales (rutas 1-190 y 400)

- Rutas periféricas (rutas 201-232)

- Microbuses y minibuses (rutas 300-340)

- Metrobus (rutas 401-420)

- Intramunicipales (rutas 501-527)

- Suburbana (rutas 51-53-601-685)

- Transmetro

Durante el censo se hizo bitácora de las rutas que transitaban en la intersección de estudio, de las cuales están: R-223, R-20, R-42, R-107, R-85, R-224, R-67, R-130, R-50, R-172, R-39, R-224, R-113, R-228, R-42, R-601, R-71 y R-31. Si comparamos las rutas que transitan en la intersección, contra las subcategorizaciones, notamos que las rutas radiales y periféricas son las que se encuentran con mayor frecuencia.

\section{RUTAS PERIFÉRICAS}

Anteriormente se conocían a las rutas periféricas como aquellas rutas que se trasladaban de un municipio a otro sin pasar por el centro de Monterrey, a excepción de las rutas 206, 209 y 214. Sin embargo, en 2004 varias rutas radiales pidieron el cambio a aumentar sus tarifas y que se les considerara como rutas periféricas, debido a que superaban el kilometraje promedio de rutas radiales que es de $30 \mathrm{Km}$. Por ello muchas rutas radiales se convirtieron en periféricas.

Hoy en día por las modificaciones de rutas radiales a periféricas en el año 2004, muchas rutas radiales que llegaban al centro siguieron el ejemplo de convertirse a periféricas (a excepción de las rutas 221 y 240):

- Ruta 220, su raíz es la ruta 56

- Ruta 222, su raíz es la ruta 122

- Ruta 223, su raíz es la ruta 93

- Ruta 224 , su raíz es la ruta 85

- Ruta 225 , su raíz es la ruta 125

- Ruta 226, su raíz es la ruta 55

- Ruta 227, su raíz es la ruta 17 
- Ruta 228, su raíz es la ruta 103

- Ruta 229, su raíz es la ruta 129

- Ruta 230, fue empezada desde 0

- Ruta 231, empezada de 0 y hoy en día está desaparecida

- Ruta 232, su raíz es la ruta 56

\section{RUTAS RADIALES}

Actualmente las rutas radiales son aquellas que transitan de un municipio a otro, cruzando por el centro de Monterrey obligatoriamente.

\section{NORMATIVAS DEL ESTADO ACTUALMENTE IMPLEMENTA- DAS}

En la Norma Técnica Estatal de Aceras de Nuevo León existen diferentes aspectos que se deben considerar al momento de implementar mobiliario urbano en banquetas, aceras y calles. Este proyecto se enfocará principalmente en la sección de paradas de autobús, rescatando de esta forma los lineamientos, premisas constructivas y dimensiones para así aplicarlas a la propuesta definitiva.

Según esta norma se deben tomar 10 consideraciones al implementar una parada de autobús en el estado de Nuevo León, las cuales son:

1. Tendrán como función brindar áreas de espera y resguardo ante el clima para los usuarios del sistema de transporte público, puede contar con una banca o con apoyos que permitan recargar la parte trasera de las personas y espacio para personas en silla de ruedas.

2. Se debe tener en cuenta al área para abordar el vehículo para personas en silla de ruedas con un ancho libre mínimo de 1.20 metros.

3. Se deben tomar en cuenta los obstáculos y la presencia de otros tipos de mobiliario urbano, y evitar obstaculizar el flujo de usuarios.

4. Las dimensiones mínimas son de 1.60 metros de ancho por 3.50 metros de largo y 2.40 metros de altura libre (Figura 3 y 4). 


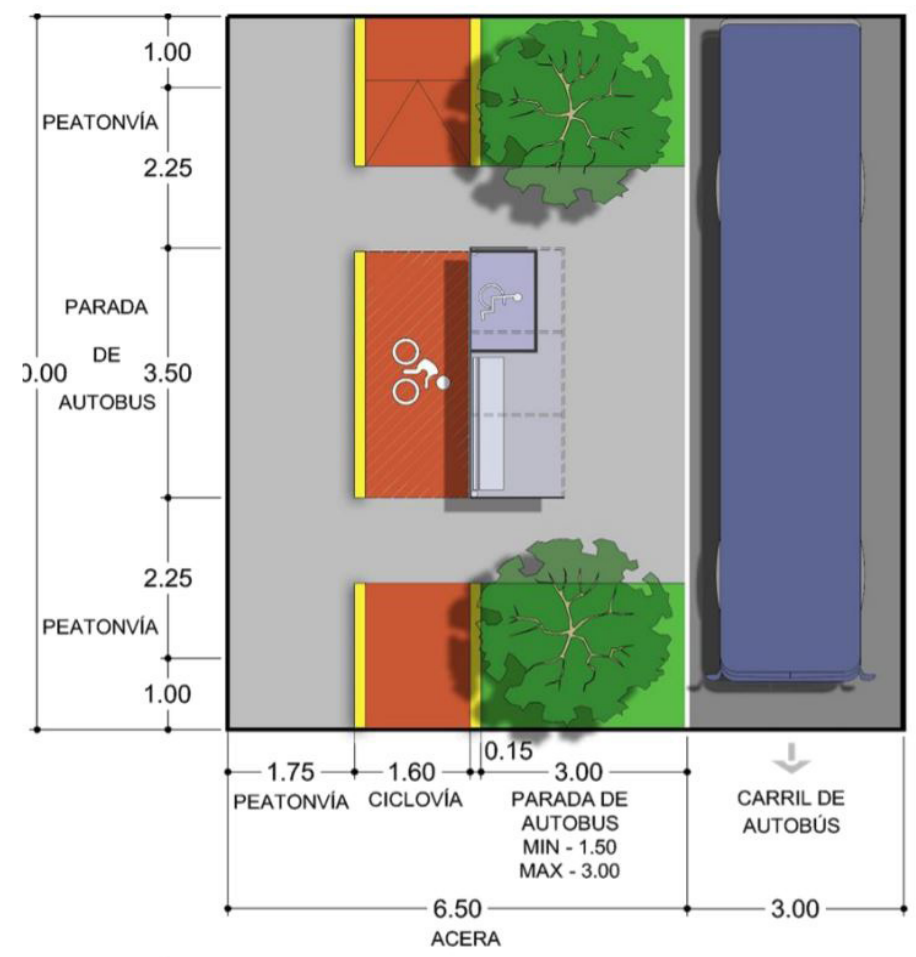

Figura 3. Dimensionamiento de aceras en vista superior. Adaptado de Dimensionamiento superior en aceras de 3.5 m. (León, 2020).

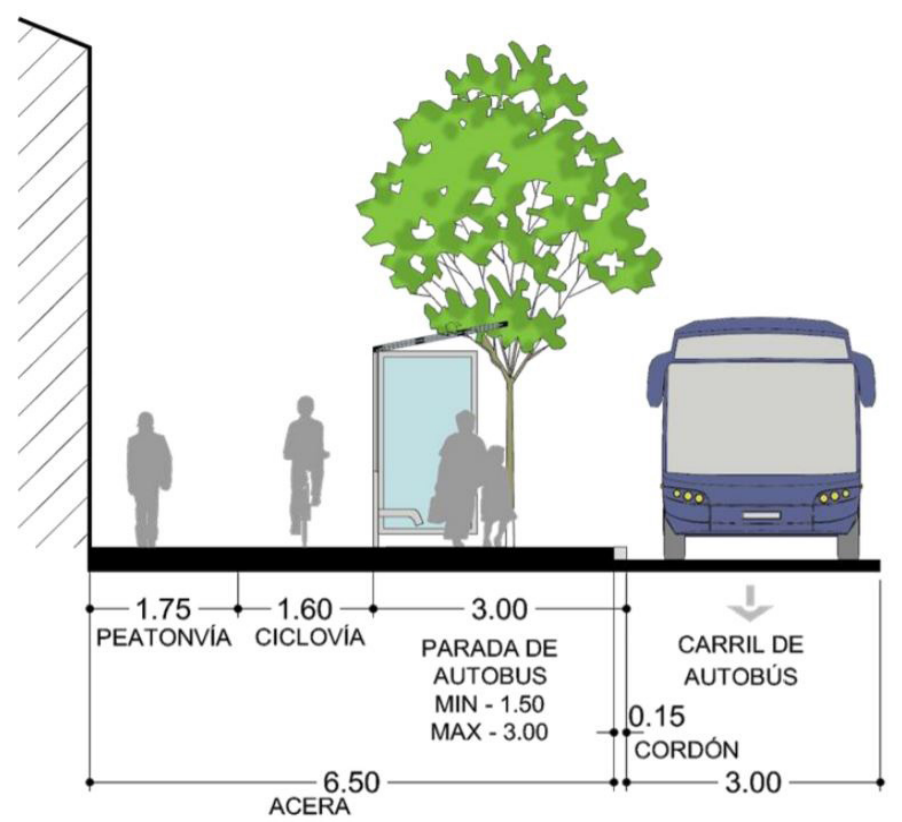

Figura 4. Dimensionamiento de aceras en vista lateral. Adaptado de Dimensionamiento lateral en aceras de 3.5 m. (León, 2020). 
5. Las paredes de las paradas de autobús deberán ser de acrílico o vidrio templado procurando la transparencia de esta.

6. Las paradas se deben ubicar en la franja de servicios sin obstaculizar la franja de la acera. En el caso de las aceras de 2.50 metros esta sección debe ser ampliada para alojar la parada de autobús.

7. La publicidad o anuncios dentro o aledaños a las paradas de autobús nunca deberán tapar la visibilidad de las personas esperando el camión, así tampoco deberán utilizarse en paredes perpendiculares al paso peatonal cerca de las paradas de autobús, procurando la transparencia de la parada (Figura 5).

8. La estructura de la parada debe ser de acero inoxidable, aluminio, plástico u otros materiales reciclados de similar durabilidad.

9. Debe tener una banca hecha de los mismos materiales permitidos para la estructura.

10. Cuando haya carril ciclista en la vía en la que se implantará la parada de autobús, se resolverá como se muestra en las figuras (León, 2020).

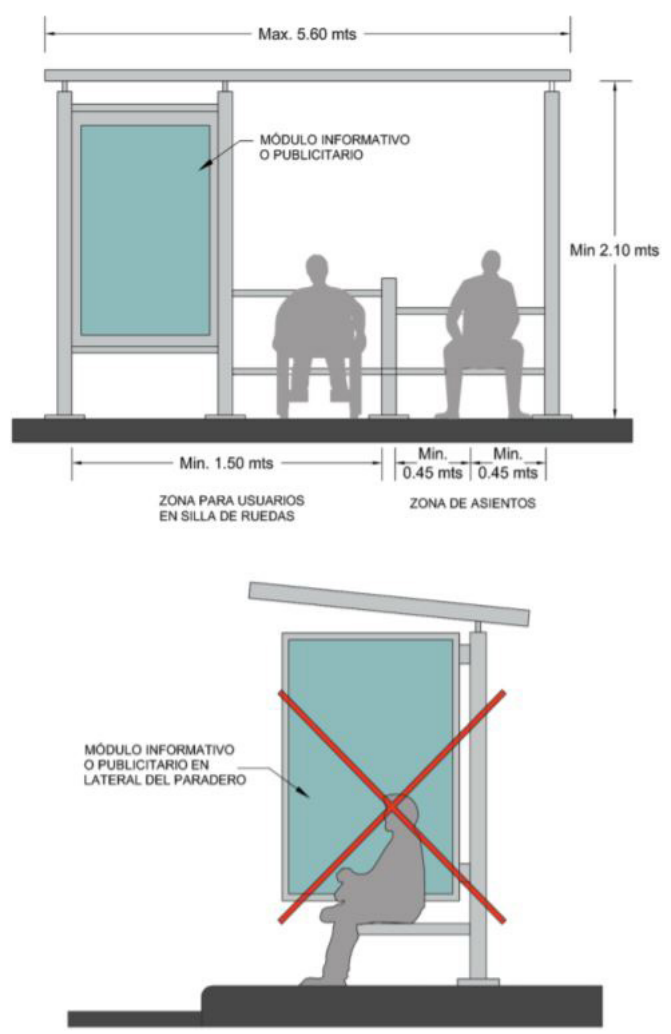

Figura 5. Colocación de publicidad en paradas de autobús. Adaptado de (León, 2020). 
Además de lo antes mencionado, existe el Manual de Normas Técnicas de Accesibilidad de la CDMX, donde especifica los parámetros a tomar en cuenta al momento de diseñar espacios públicos, edificaciones, áreas de servicio y lineamientos del diseño universal. Esto ayuda a la creación del estudio de sitio y aporta nuevas ideas a la propuesta preliminar de la parada de autobús.

\section{FASES 3, 4, Y 5: DEFINIENDO LA PROBLEMÁTICA, ANÁLISIS DE DATOS Y CARACTERÍSTICAS DEL DISEÑO.}

Se utilizó la herramienta de diseño basada en el contexto llamado "Generación de ideas orientadvvas a los ecosistemas" propuesta por la Dra. Sofía Luna y el LDI. Gustavo Zepeda. Esta ayudó para identificar características e insights de diseño.

Esta generación de ideas es un método de investigación que auxilia al diseño basado en el usuario para la recolección y síntesis de información, influenciada por las situaciones que se generan en el contexto. Esto para complementar el desarrollo del producto, en este caso la parada de autobús. Implementa el pensamiento del ecosistema por medio del ser (Luna, 2018):

- Ecosistémico: los diseñadores identifican e investigan acerca de los diferentes contextos involucrados en el desarrollo de productos.

- Dirigido por el contexto: los diseñadores definen las interacciones entre los contextos y sus componentes.

- Centrado en el ser humano: los diseñadores relacionan las interacciones humanas con otros elementos del contexto. La generación de Insight ecosistémico fortalece la claridad estratégica de los equipos de diseño al aportar holismo, flexibilidad y apertura en la fase de investigación del proceso de diseño basado en el usuario.

\section{PLANTILLA PARA LA GENERACIÓN DE IDEAS ORIENTADAS A LOS ECOSISTEMAS}

- Esta herramienta utiliza 4 contextos generales: contexto de producto, sociocultural, ambiental y contexto extendido (Figura 6). 
- Contexto del producto: es donde se encontrarán y definirán las características e insights del producto, mejoras, oportunidades, experiencias, definición de sistemas, estrategias, materiales, funciones y estética.

- Contexto sociocultural: se consideran las necesidades humanas, emociones, rutinas; la importancia de los valores, tradiciones; además de especificaciones de género, creencias, salud y bienestar. Toda esta información es fundamentada con anterioridad gracias a información ya antes recolectada (entrevistas, estudios de caso, observación).

- Contexto ambiental: es el contexto nativo del espacio, todo lo natural y lo construido de esta área específica, incluyendo su geografía, clima y recursos, infraestructura, objetos, transporte, instalaciones y características.

- Contexto ampliado: se enfoca principalmente en la futurología y prospectiva del lugar según los estudios sociales, megatendencias, que son las corrientes o acontecimientos que trascienden en la toma de decisiones de consumidores, empresas y gobierno, y observación. La economía, la innovación, tecnología, sostenibilidad, política, reglamentos, seguridad, energía, logística, desarrollo empresarial son solo algunos de los puntos que se pueden tomar en cuenta en esta sección.

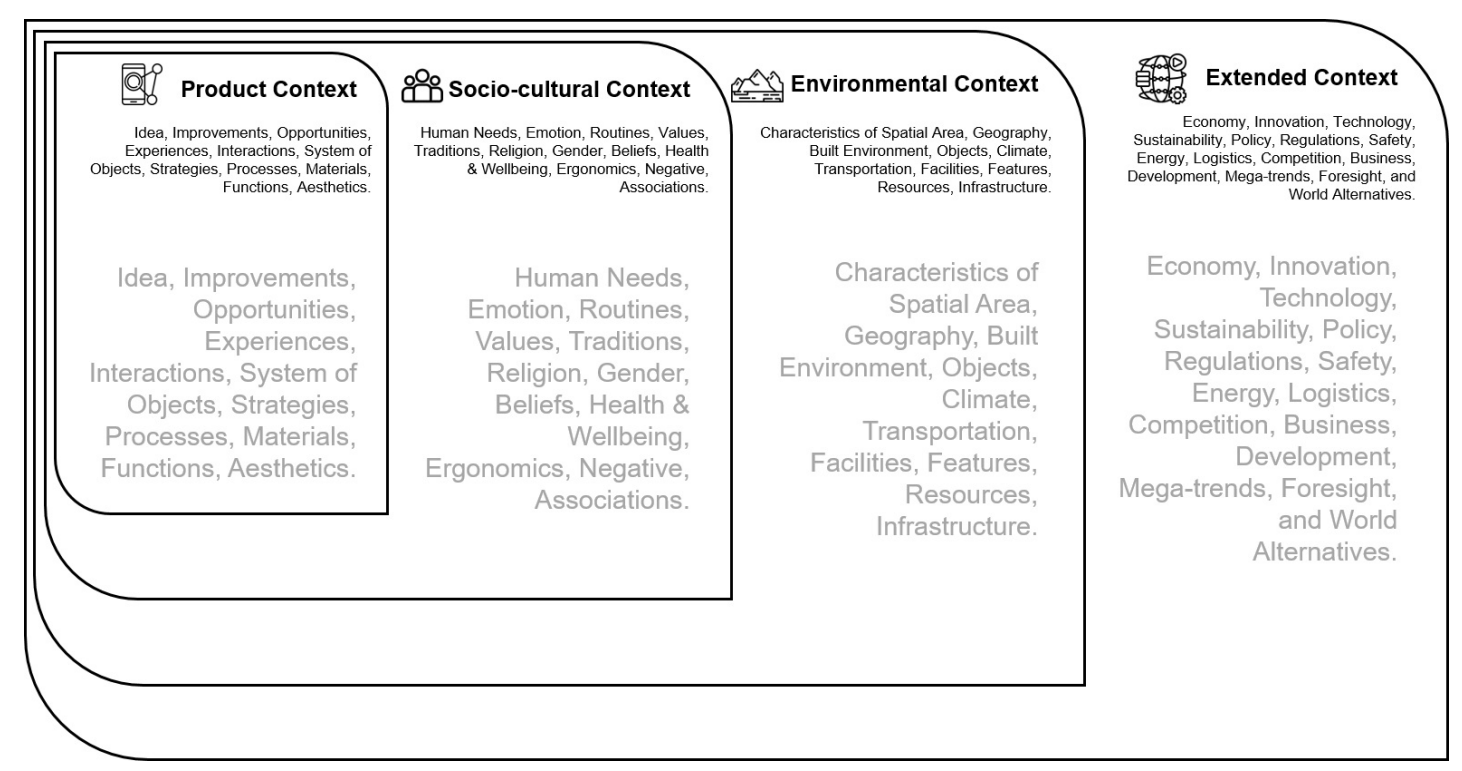

Figura 6. Tabla para generar ración de ideas sobre ecosistemas. Adaptado por Godino (2019) 


\section{PROCEDIMIENTO DE EJECUCIÓN DE LA METODOLOGÍA DE DISEÑO}

Los pasos para utilizar la Plantilla de Generación de Ideas Orientadas a los Ecosistemas variarán según el problema de diseño, en este caso los pasos para la ejecución fueron los siguientes:

1. Se realizó la plantilla sobre una superficie plana, siguiendo el formato anteriormente descrito (Figura 7).

2. Después se definió un color para cada sección; azul para el contexto extendido, rosa para el contexto ambiental, anaranjado para contexto sociocultural y blanco para contexto de producto y características. Además se definieron periodos de 5 minutos para la generación de ideas y 10 minutos de reposo para después tener otra sesión de ideas (Figura 8).

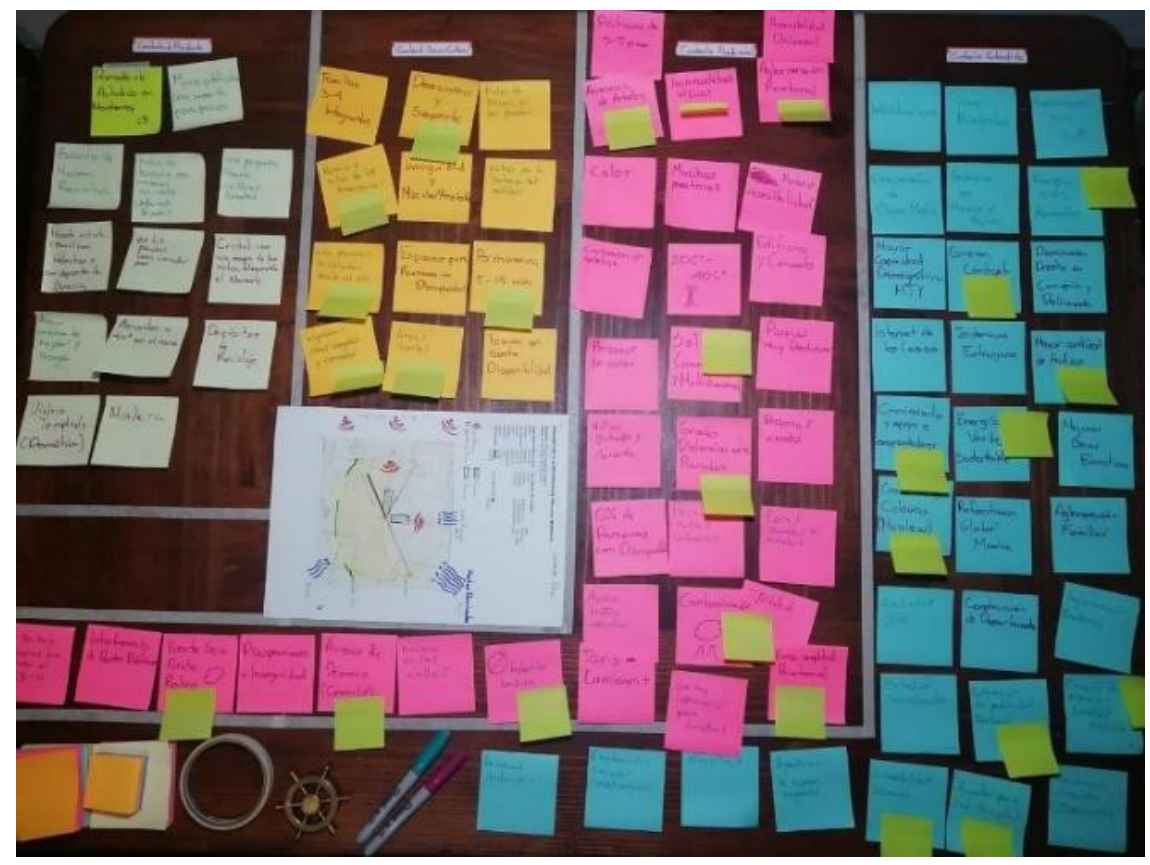

Figura 7. Técnica de Generación de Ideas Orientadas a los Ecosistemas. Adaptado por Godino (2019)

3. Todas las ideas, insights sobre megatendencias y prospecciones de la Cd. de Monterrey, se posicionaron con post-it en la sección de Contexto Ampliado. 
4. Posteriormente y tomando los mismos periodos de tiempo asignados se siguió con el contexto ambiental; aquí cabe señalar que se utilizaron herramientas de análisis externas, como es el análisis de sitio de la intersección (Figura 8) y los resultados del censo vehicular enfocado en camiones urbanos.

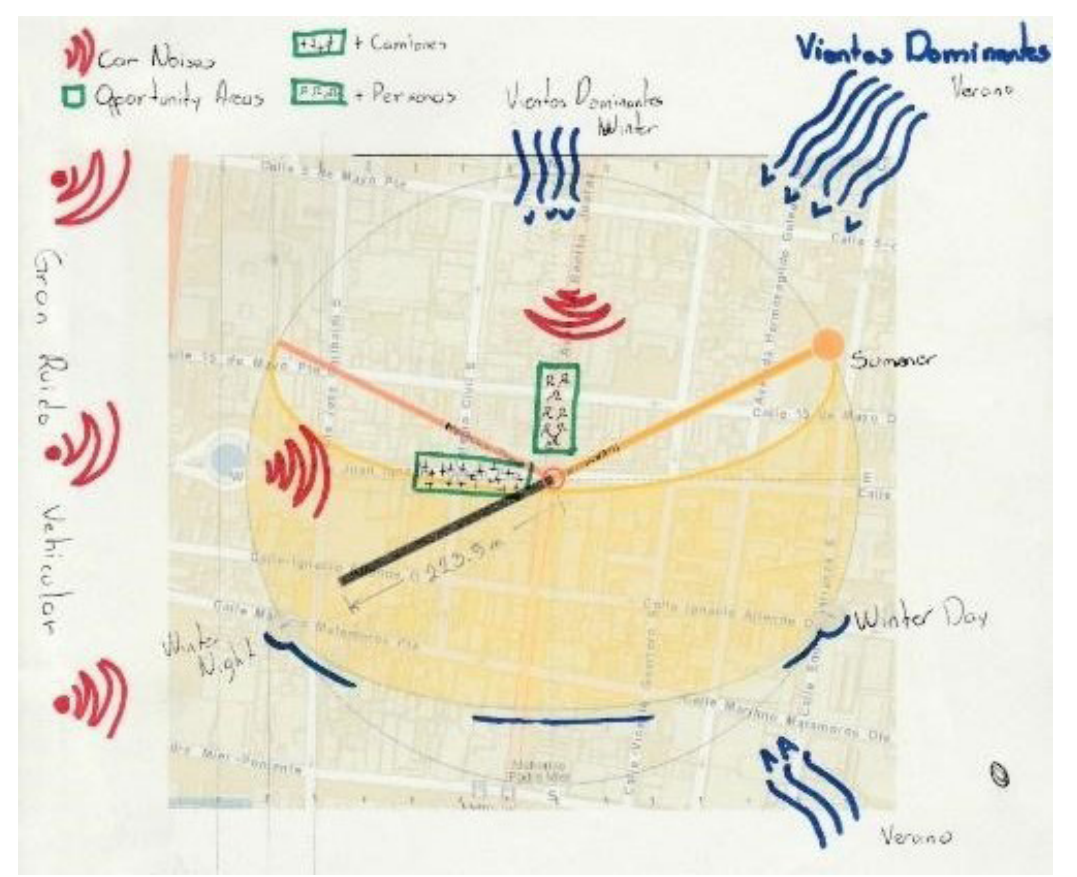

Figura 8. Análisis de sitio de intersección Calle Benito Juárez y Calle Juan I. Ramón. Adaptado por Godino (2019)

5. En cuanto al apartado de contexto sociocultural se hizo un mayor enfoque a dos fundamentos; el primero es de las emociones experimentadas y observadas por los ciudadanos y usuarios de los camiones urbanos que se observaron al momento de hacer el censo vehicular, y el segundo es la percepción que la sociedad quisiera sentir al momento de utilizar una parada de autobús.

6. a una vez teniendo estos 3 contextos completos y con sus respectivas ideas, características e insights, se inició la tarea de realizar la técnica de “Relaciones Forzadas”. Se tomaron los insights más realistas de cada apartado, señalándolos con pequeños post-it color amarillo; luego, ya 
categorizado cada apartado, se tomaba un insight de cada contexto, para luego forzarlos en un conjunto lógico y posicionarlos en la sección de Contexto de Producto; se toma esta como una característica de diseño.

\section{CARACTERÍSTICAS DE DISEÑO}

Con base en la sesión creativa anteriormente mencionada se recopiló la siguiente información (Tabla 1).

\begin{tabular}{|c|c|c|}
\hline Contexto extendido & Contextoambiental & Contexto sociocultural \\
\hline Energía $100 \%$ renovable & Ausencia de árboles & $\begin{array}{c}\text { Permanencia en paradas de 5-15 } \\
\text { min }\end{array}$ \\
\hline Green Context & Incomodidad visual & Necesidad de espacios amplios \\
\hline Mayor cantidad de autos & Aglomeración peatonal & \\
\hline $6 s 4$ se & Sol constante & \\
\hline Crecimiento a emprendedores & Grandes distancias entre paradas & \\
\hline Energía verde sustentable & Contaminación & \\
\hline Ciclovías & No existen botes de basura & \\
\hline Publicidad peatonal & Ausencia de áreas de descanso & \\
\hline Ayuda a turistas & Viento seco & \\
\hline Accesibilidad universal & & \\
\hline
\end{tabular}

Tabla 1. Características del diseño según su contexto. Adaptado por Godino (2020)

Los insights recopilados fueron mezclados entre ellos con el objetivo de lograr una relación forzada y conseguir una característica específica que pueda tener el diseño definitivo de la parada de autobús. Además, también se consideraron las megatendencias de individualidad y conectividad para crear un diseño completo.

La megatendencia hacia la conectividad indica que en todas las áreas de la vida, tanto en lo personal como en lo profesional, las personas estarán interconectadas entre sí a través de los dispositivos inteligentes que se conectan entre ellos por medio de Internet y que permiten a las personas comunicarse, 
convirtiéndose en compañeros inseparables en la vida cotidiana. En este mundo digital vinculado, los servicios futuros deberán ser integrales y discretos. El servicio futuro debe ajustarse a las necesidades específicas de cada persona, pero todo tiene que funcionar a un nivel más alto. Este servicio meta ha sido posible gracias a la digitalización amplia en todos los ámbitos de la vida. Debido al cambio de enfoque del grupo objetivo para el consumo situacional, el cliente ya no es simplemente un consumidor, sino que también se convierte en un emisor que envía los requisitos necesarios en un determinado momento, dando lugar a la mega tendencia de individualidad (Bustos, 2020).

\section{FASE 6: IDEACIÓN E ITERACIÓN DE DISEÑO}

Después de haber analizado la información planteada en la generación de ideas orientadas a los ecosistemas se prosiguió a idear las características del diseño (Tabla 2).

\begin{tabular}{|l|l|}
\hline Muro publicitario con soporte para brazos & Asientos de $45^{\circ}$ en el muro \\
\hline Asiento de madera reciclada & Depósitos de reciclaje \\
\hline $\begin{array}{l}\text { Bote de basura con material } \\
\text { reciclado }\end{array}$ & Vidrio templado \\
\hline Pequeña librería con libros donados & Materiales innovadores \\
\hline $\begin{array}{l}\text { Parada ciclista con helechos o depósitos de } \\
\text { basura }\end{array}$ & $\begin{array}{l}\text { Cristal con mapa de rutas, bloqueando } \\
\text { el noreste }\end{array}$ \\
\hline
\end{tabular}

Tabla 2. Insights de diseño para muros publicitarios y asientos en paradas de autobús. Adaptado por Godino (2020)

Con estas características se comenzaron a explorar formas por medio de bocetos (Figura 9), implementando cada una de las propuestas encontradas. Se hicieron combinaciones entre ellas y se llevaron a una propuesta más formal y definitiva. De igual forma, a cada propuesta se le dio un código de color para poder identificarlas como tales y no llegar a confusiones futuras y así poder hacer futuras valuaciones. 


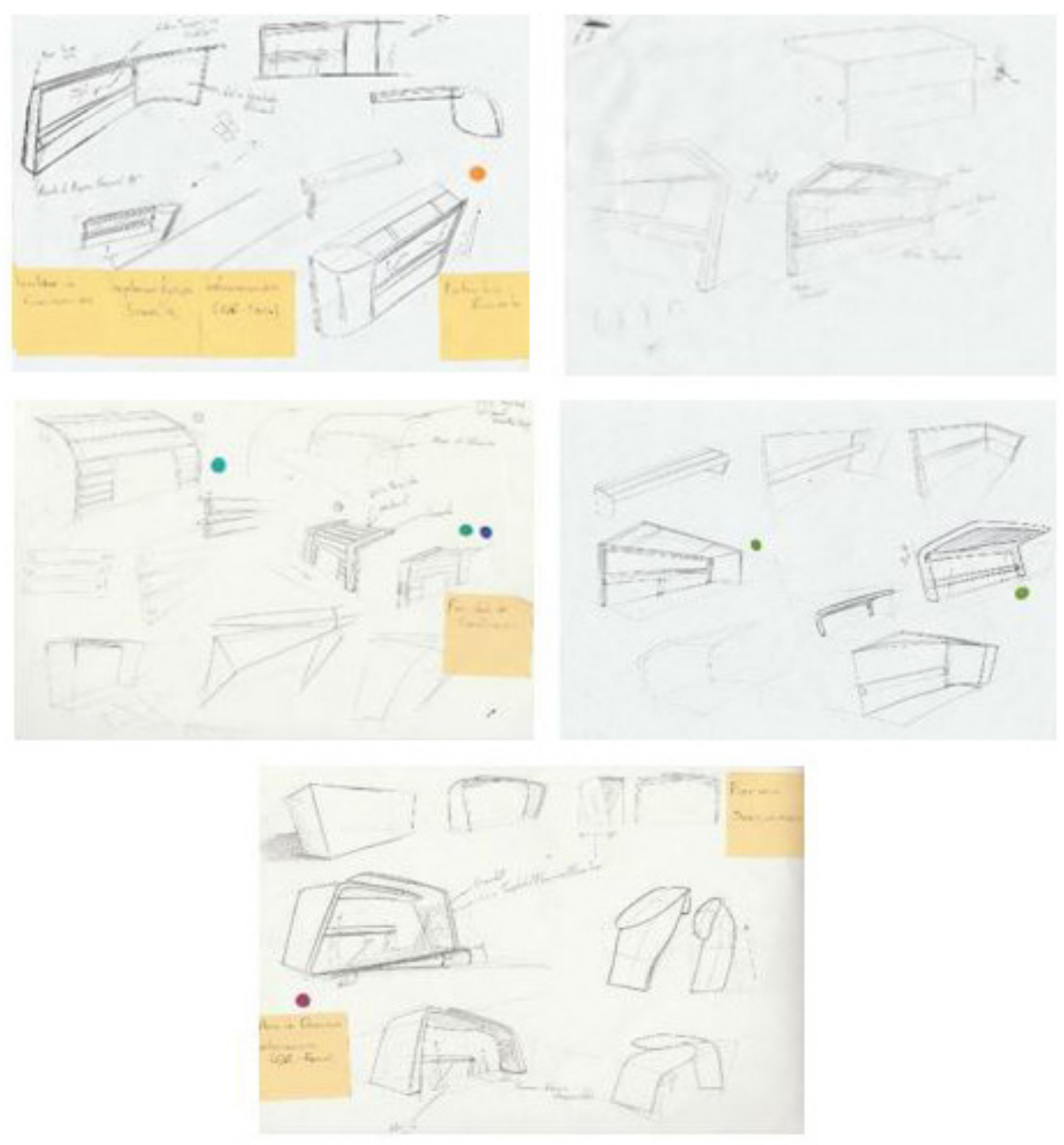

Figura 9. Exploración de formas. Adaptado por Godino (2020)

Teniendo diversas propuestas con las características indicadas se pasó a realizar una matriz de polaridades entre todas las propuestas (Figura 10). Lo que busca esta técnica de validación es enlistar las necesidades y características que debe tener el diseño final (funcionalidad, manufactura, seguridad, rentabilidad, etc.) para así compararlas y llegar a la más viable según sus atributos (Haberfellner, 1972). 


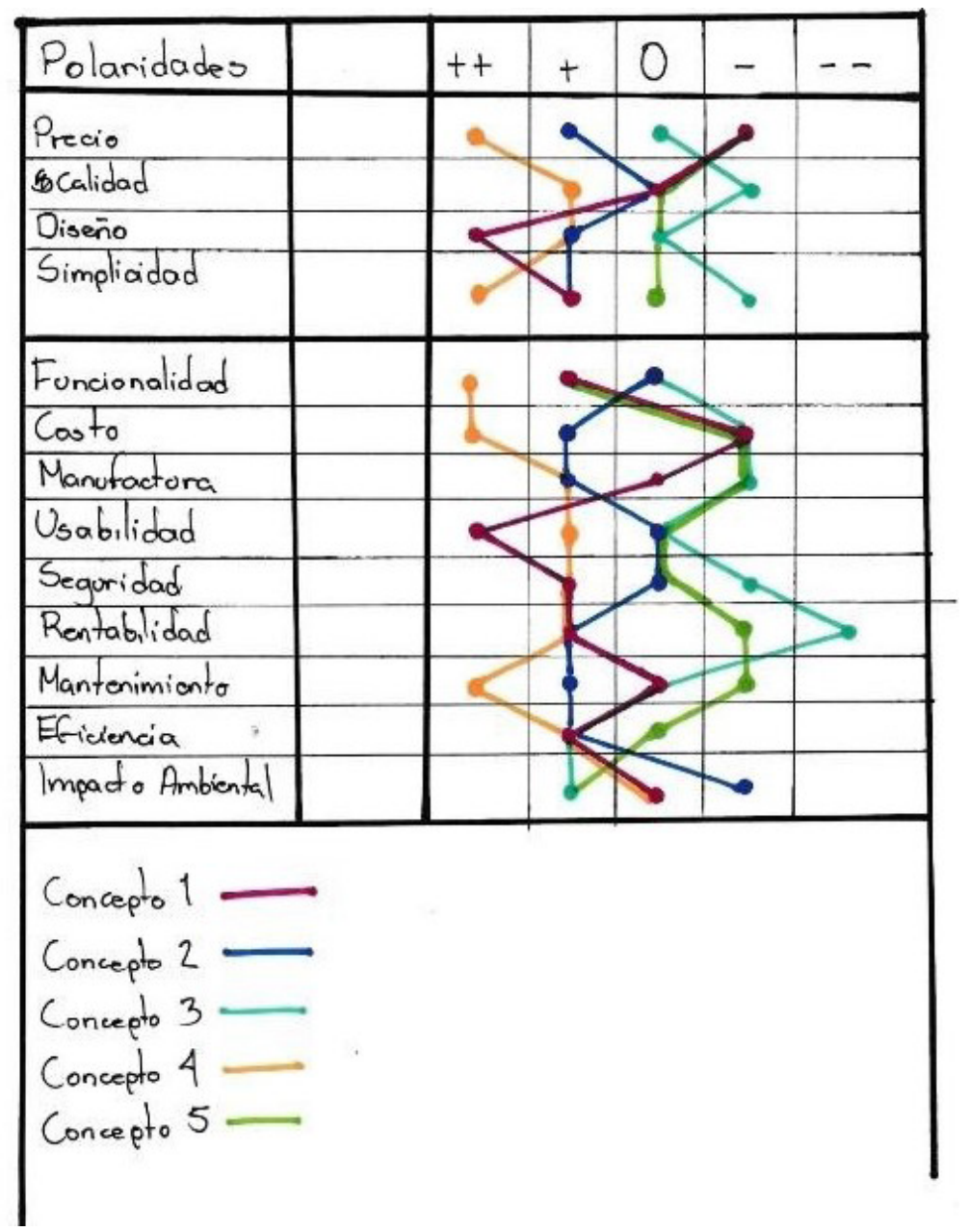

Figura 10. Matriz de polaridades de propuestas de diseño. Adaptado por Godino (2020)

Cada una de las propuestas fue analizada por medio de distintas valoraciones para poder llegar al concepto que cumpliera, dentro de los contextos, con las características y objetivos encontrados en cada fase. Después de analizar cada propuesta con los parámetros que deberían abarcar, como se mostró en la matriz de polaridades, se concluyó que el concepto 4 sería el mejor candidato como producto definitivo (Figura 11). Este concepto cuenta con un muro publicitario con soporte para brazos, asientos de $45^{\circ}$ en la estructura y cristal con mapa de rutas, bloqueando el noreste. Se trata de unas de las características de diseño planteadas a partir de la generación de ideas orientadas a los ecosistemas y cumpliendo con la Norma Técnica Estatal de Aceras de Nuevo León, con lo que permite una mejor interacción entre el usuario y el entorno. 


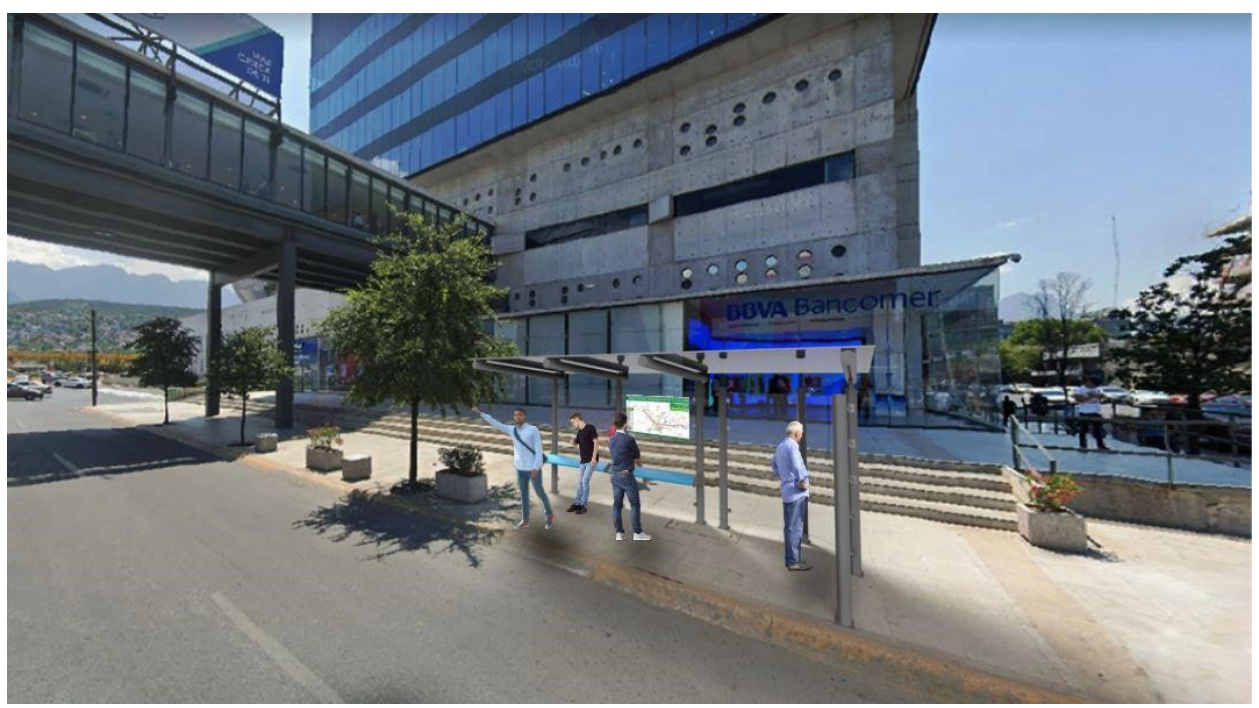

Figura 11. Propuesta de diseño definitiva. Adaptado por Godino (2020).

\section{CONCLUSIÓN}

La investigación sobre un enfoque ecocéntrico ayuda a identificar con mayor precisión cuáles son los factores que influyen en el cambio de ambientes externos al diseño que el antropocentrismo no puede llegar a percibir. Hay que revisar a profundidad lo que rodeará nuestra estrategia de comunicación, nuestro producto o nuestro sistema de innovación para poder esclarecer con mayor relevancia la función y estética del diseño general.

El desarrollo del diseño Contexto-Entorno con base en tres variables: reformar el diseño contextual al diseño por contexto, introducir el punto de vista ecocéntrico dejando de lado el antropocéntrico y aplicar la autopoiesis de diseño para lograr esto, puede apoyar al desarrollo de una filosofía que se explora como metodología aplicable en métodos de diseño, para lograr comprender el nuevo panorama definido por estas tres variables.

Podemos considerar que las metodologías ecocentristas generarán un mayor impacto en la percepción del diseño para los usuarios, a través de una nueva forma de generar nuevas tendencias y desarrollar las ya existentes. Esto debido a que es funcional y, por tanto, puede ser factible para distintos enfoques, desde diseño de productos y servicios, hasta sistemas y aspectos tecnológicos. También permite la posibilidad de hacer más holística la investigación contextual, tomando en consideración la posible influencia del uso 
del Contexto-Entorno en otras áreas del diseño, como también el explotar la teoría de sistemas ecológicos hacia la relación entre el entorno y los objetos definiendo así una autopoiesis mucho mayor relacionada con los objetos.

El individualismo no dejará de tener importancia dentro de estos nuevos métodos, ya que esta macrotendencia es la que rige el diseño del siglo XXI. Sin embargo, complementar con una visión más allá del usuario hará, y queda claro, un diseño completo por definición.

\section{REFERENCIAS}

Alexander, C. (1964). Notes on the Synthesis of Form. Cambridge: University Press.

Bustos, M. (2020). Las Mega-tendencias para la década del 2020. Recuperado de: https://www.elevate.com.mx/las-mega-tendencias-para-la-decada-del-2020/

Godino, F. (2019). Implementación de Concreto Fotoluminiscente en entornos urbanos y la influencia del ecocentrismo en el diseño industrial (tesis de pregrado). Universidad Autónoma de Nuevo León, Monterrey, México.

Haberfellner, R. (1972). Systems Engineering: Fundamentals and Applications. Basilea: Birkhauser.

León, G. (2020). Norma Técnica Estatal de Aceras de Nuevo León. Monterrey, Nuevo León, México.

Luna, S. (2018). Generación de conocimiento de los ecosistemas: Innovación de la investigación impulsada por el contexto dentro del proceso de diseño centrado en el ser humano. Monterrey, Nuevo León, México.

Oxman, N. (2016). Towards a material ecology. World Economic Forum. Recuperado de: http://www.weforum.org/agenda/2016/01/towards-a-material-ecology

Wong W. (2002). Fundamentos del diseño bidimensional y tridimensional, Barcelona: Editorial Gustavo Gili. 
Zepeda, G. (2017). Diseño Contexto-Entorno: Diseño por contexto a través del ecocentrismo para su aplicación industrial, Memorias del Seminario Internacional de Investigación en Diseño, 8, 654-663. 